\title{
Children at risk of developing antisocial behaviours show deficits in affective empathy
}

\section{By Dr Jessica Edwards}

Researchers in the Netherlands and UK have monitored cardiovascular and electrodermal activity and eye tracking to assess affective and cognitive empathy in children at high risk of engaging in criminal behaviours. Children composing the high-risk group (114 boys aged 8-12 years) were recruited through the ongoing "Preventive Intervention Trajectory" study of younger siblings or children "of delinquents" and children failing primary school in Amsterdam, Netherlands. The high risk children, and a group of typically developing controls, were shown four different video clips: one neutral clip and three clips aiming to elicit emotions of fear, happiness or pain. During each clip, visual fixation to the video screen and attention to socially relevant cues (namely the eyes and faces of the actors) was determined by eye tracking and used as an indicator of social attention. Following the clips, the participants were asked to identify the type of emotion portrayed and its intensity to determine cognitive empathy. The researchers found no marked differences in these two parameters between the high risk and control groups. Heart rate and the skin conductance response (SCR) was also recorded during the presentation of each clip and used as a measure of affective empathy. Here, the high risk group had a lower heart rate when observing clips of pain and fear, and reduced SCR when observing pain. The researchers propose that specific deficits in affective empathy for negative emotions may have an important role in antisocial behaviour development. Going forward, they suggest that interventions should target affective empathy to promote pro-social behaviour.

Referring to:

van Zonneveld, L., Platje, E., de Sonneville, L., van Goozen, S. \& Swaab, H. (2017), Affective empathy, cognitive empathy and social attention in children at high risk of criminal behaviour. J Child Psychol Psychiatr. 58: 913-921. doi:10.1111/jcpp.12724
Glossary:

Cognitive empathy: the capacity of an individual to understand what others' emotions and thoughts might be, without being emotionally involved.

Affective empathy: the capacity of an individual to experience another person's feelings while understanding that they are distinct from one's own feelings.

Social attention: attention to socially relevant cues, in particular facially expressed cues, to gain information about the mental and emotional state of another person during social interaction.

Skin conductance response (SCR): a phenomenon whereby the skin momentarily becomes a better conductor of electricity when either external or internal stimuli occur that are physiologically arousing. Thus, the SCR is an objective, transient indication of autonomic nervous system arousal in response to a stimulus.

Pro-social behaviour: engagement in behaviours and/or acts that are considered to be generally beneficial or intended to help other people. Pro-social behaviours include feeling empathy or concern for others. 